\title{
A Novel Approach to the Fabrication of CdSe Quantum Dots in Aqueous Solution: Procedures for Controlling Size, Fluorescence Intensity, and Stability over Time
}

\author{
M. J. Almendral-Parra, ${ }^{1}$ A. Alonso-Mateos, ${ }^{1}$ J. F. Boyero-Benito, ${ }^{1}$ \\ S. Sánchez-Paradinas, ${ }^{1}$ and E. Rodríguez-Fernández ${ }^{2}$ \\ ${ }^{1}$ Departamento de Química Analítica, Nutrición y Bromatología, Facultad de Ciencias Químicas, Universidad de Salamanca, \\ Plaza de la Merced, s/n, 37007 Salamanca, Spain \\ ${ }^{2}$ Departamento de Química Inorgánica, Facultad de Ciencias Químicas, Universidad de Salamanca, \\ Plaza de la Merced, s/n, 37007 Salamanca, Spain
}

Correspondence should be addressed to M. J. Almendral-Parra; almendral@usal.es

Received 10 October 2013; Accepted 4 April 2014; Published 24 April 2014

Academic Editor: M. Ghoranneviss

Copyright (C) 2014 M. J. Almendral-Parra et al. This is an open access article distributed under the Creative Commons Attribution License, which permits unrestricted use, distribution, and reproduction in any medium, provided the original work is properly cited.

\begin{abstract}
This paper report a straightforward approach for the synthesis of CdSe quantum dots (CdSe QDs) in aqueous solution. This method, performed in homogeneous phase, affords optimal sizes and high quantum yields for each application desired. It is an $a$ la carte procedure for the synthesis of nanoparticles aimed at their later application. By controlling the experimental conditions, CdSe QDs of sizes ranging between 2 and $6 \mathrm{~nm}$ can be obtained. The best results were achieved in an ice-bath thermostated at $4^{\circ} \mathrm{C}$, using mercaptoacetic acid as dispersant. Under these conditions, a slow growth of quantum nanocrystals was generated and this was controlled kinetically by the hydrolysis of $\mathrm{SeSO}_{3}{ }^{2-}$ to generate $\mathrm{Se}^{2-}$ in situ, one of the forming species of the nanocrystal. The organic dispersant mercaptoacetate covalently binds to the $\mathrm{Cd}^{2+}$ ion, modifying the diffusion rate of the cation, and plays a key role in the stabilization of CdSe QDs. In optimum conditions, when kept in their own solution CdSe QDs remain dispersed over 4 months. The NPs obtained under optimal conditions show high fluorescence, which is a great advantage as regards their applications. The quantum efficiency is also high, owing to the formation under certain conditions of a nanoshell of $\mathrm{Cd}(\mathrm{OH})_{2}$, values of $60 \%$ being reached.
\end{abstract}

\section{Introduction}

Quantum dots (QDs) are semiconductor nanoparticles (NPs) that have recently attracted much interest in biological research because of their unique spectral properties [1-3]. They are composed of elements from groups II-VI, III-V, and IV-VI of the periodic table [4], having a more or less spherical shape and sizes typically ranging from 1 to $12 \mathrm{~nm}$. They exhibit unique optical and electronic properties based on a strong quantum-confinement effect $[5,6]$. One of the extraordinary properties of QDs is that their particle size determines most of their properties, mainly the wavelength of fluorescence emission, which can be altered by manipulating their size [7]. In comparison with traditional organic fluorophores, QDs have considerable advantages [8], including size-dependent emission, narrow emission bands, and high luminescence. Furthermore, the high surface/volume ratio of QDs allows them to be used for the design of more complex nanosystems [9]. All the above properties make QDs good fluorescent markers for biological and biomedical applications, in particular in cellular imaging [10]. Moreover, they have attracted great interest for their potential application in electronic and optoelectronic devices [11, 12]. Strongly luminescent semiconductor nanocrystals are highly desirable for a large number of optoelectronic applications, such as light-emitting diodes (LEDs) [13]. In recent years, intense efforts have been made in the field of the synthesis and use of photoluminescent QDs as markers for biochemical applications in 
bioanalysis, diagnosis, and in in vivo imaging. The different requirements in each case have resulted in the development of many different synthesis procedures, widely reported in the literature. Among the different semiconductor NPs, the synthesis of CdSe nanocrystals continues to be the field most widely investigated, mainly because the method of preparation used affords an exceptional degree of control over the size and shape of the nanocrystals obtained. High quality QDs are usually prepared at elevated temperatures in organic solvents such as tri- $n$-octylphosphine oxide (TOPO) or a mixture of TOPO and tri- $n$-octylphosphine (TOP). The surfaces of the QDs prepared in an organic phase are coated with a hydrophobic layer of TOP or TOPO molecules. However, these QDs cannot be applied directly in biosystems owing to the organic nature of their surfaces. Normally, it is necessary to replace the molecules coating the surface of the nanoparticle, using time-consuming methods of ligand exchange that significantly reduce the quantum yield of QDs. Additionally, it has been found that after ligand exchange these hydrophilic QDs are not sufficiently stable in aqueous solution. Some techniques of surface modification aimed at increasing the hydrophilic character and stability of QDs in aqueous solution have also been used. This change has often provided a significant increase in the diameter of the QDs to close to $20-30 \mathrm{~nm}$. In contrast to organic synthesis, aqueous synthesis exhibits good reproducibility and low toxicity, and it is cheap, and-in particular-the products thus prepared have excellent water solubility, stability, and biological compatibility. Despite this, QDs prepared in aqueous phase tend to have low quantum yields (QY: 3-10\%). However, perhaps owing to excessive haste in obtaining practical results that would meet the high expectations that will undoubtedly arise in relation to the use of QDs in biological media, our knowledge of their structures and chemical behavior (both of them critical aspects for optimizing the bioconjugation process) remains somewhat shallow. However, in recent years, methods for preparing luminescent QDs in aqueous solution have increased substantially in number due to the work of Zhang et al. [13, 14], who have made considerable advances in improving QY. All the results show that synthesis processes in aqueous media are an attractive alternative to the synthesis of QDs in organic media and they are now an important avenue of enquiry.

In the present work we report a simple procedure for the preparation of CdSe QDs in aqueous solution. By controlling the experimental conditions, CdSe QDs of sizes ranging between 2 and $6 \mathrm{~nm}$ can be obtained. The best results were achieved in an ice-bath thermostated at $4^{\circ} \mathrm{C}$, using mercaptoacetic acid as dispersant. Under these conditions, a slow growth of quantum nanocrystals was generated and this was controlled kinetically by the hydrolysis of $\mathrm{SeSO}_{3}{ }^{2-}$ to generate $\mathrm{Se}^{2-}$ in situ, one of the forming species of the nanocrystal. This homogeneous phase synthesis allows the different variables involved in the process to be controlled in order to obtain optimum sizes and high quantum yields for each application. It is an a la carte procedure for the synthesis of the NPs addressed here as regards their future use. The QDs obtained are stable for more than three months under the experimental conditions used, maintaining their high fluorescence. NPs have been characterized by absorption spectroscopy, fluorescence spectroscopy, and transmission electron microscopy (TEM). These techniques have allowed in-depth studies of all the variables affecting their size and properties in aqueous solution for their possible use as biomarkers and in other applications of interest.

\section{Experimental}

2.1. Reagents. All chemical reagents were of analytical grade and used as purchased. Solutions were prepared with ultrahigh quality deionized water. Cadmium chloride solutions were prepared by direct weighing of $99 \%$ pure anhydrous $\mathrm{CdCl}_{2}$ (Acros Organics) and dissolution in water. Aqueous solutions of anhydrous sodium sulphite, $\mathrm{Na}_{2} \mathrm{SO}_{3}$ (Acros Organics), at different concentrations, were prepared at the time of use. Selenium powder, $99.99 \%$ pure (Aldrich), and $1 \mathrm{M}$ sodium hydroxide, $\mathrm{NaOH}$ (Scharlau), were included. $10^{-5} \mathrm{M}$ quinine sulphate solution (Acros Organics) was prepared by direct weighing and dissolution in $0.5 \mathrm{M} \mathrm{H}_{2} \mathrm{SO}_{4}$. Buffer solutions of $\mathrm{pH}=4.00$ and $\mathrm{pH}=7.00$ (Scharlau). Mercaptoacetic acid (MAA), $\mathrm{HSCH}_{2} \mathrm{COOH}, 98 \%$ (Acros Organics) was included.

2.2. Instrumentation. Fluorescence spectra were measured using a Shimadzu Model RF-5000 spectrofluorophotometer, with a Model DR-15 controller unit and a $150 \mathrm{~W}$ Xenon lamp as a light source. The slits for the excitation and emission widths were both $3 \mathrm{~nm}$. The UV-visible absorption measurements of the samples were performed on a Shimadzu UV/Vis160 spectrophotometer. The $\mathrm{pH}$ value of the solutions was measured with a Crison $501 \mathrm{pHmeter}$. TEM images were performed on a ZEISS EM-900 device.

\subsection{Synthesis of CdSe Quantum Dots in Aqueous Medium}

2.3.1. Preparation and Determination of the Concentration of Sodium Selenosulphate Solutions. As the $\mathrm{Se}^{2-}$ ion source, solutions of selenosulphate prepared from selenium powder and sodium sulphite were used and were prepared as follows. Briefly, $2.5 \mathrm{~g}$ of $\mathrm{Na}_{2} \mathrm{SO}_{3}$ was added to $100 \mathrm{~mL}$ of deoxygenated water. Then, $0.4 \mathrm{~g}$ of selenium powder was added, allowing the reaction to take place at $80^{\circ} \mathrm{C}$ in sealed containers overnight. After this time, the solution was filtered and a reddish working stock solution was obtained. The final concentration of $\mathrm{SeSO}_{3}{ }^{2-}$ was determined gravimetrically by weighing the solid selenium precipitated in acid medium. The stability of the $\mathrm{SeSO}_{3}{ }^{2-}$ solutions over time was found to persist for at least one month, their concentrations remaining constant. In the experiments carried out during this work, when the $\mathrm{SeSO}_{3}{ }^{2-}$ solution reached one month of life, a new solution was prepared and its concentration was determined.

2.3.2. General Method for the Synthesis of CdSe NPs in Aqueous Medium. The general procedure for obtaining CdSe nanoparticles in aqueous medium, without specifying the values of the variables set in each experimental study, 


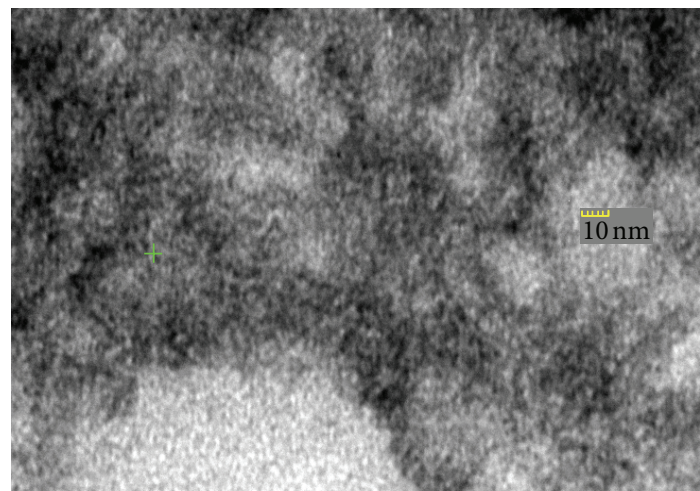

FIgURE 1: Characteristic TEM image of CdSe nanoparticles.

employed the following steps: $100 \mathrm{~mL}$ of a $\mathrm{Cd}^{2+}$ ion solution was placed in a topaz flask. Under stirring, the desired amount of a solution of 1.14 M mercaptoacetic acid was added. Then, the $\mathrm{pH}$ of the solution was adjusted with $1 \mathrm{M} \mathrm{NaOH}$. When it was necessary to work at temperatures higher than the ambient temperature, this was achieved by controlled heating. Temperatures lower than ambient temperature were achieved by introducing the flask with the solution in an icebath $\left(4^{\circ} \mathrm{C}\right)$ and keeping it there during the procedure. After the working temperature had been reached and $\mathrm{N}_{2}$ had been bubbled through the solution, the desired amount of $\mathrm{SeSO}_{3}{ }^{2-}$ was added slowly, with stirring, to control the concentration of $\mathrm{Se}^{2-}$ in solution and to avoid supersaturation. The time of the addition of this reagent ranged between 8 and $10 \mathrm{~min}$, considering this moment as $t=0$ for the kinetic studies. The final $\mathrm{pH}$ was checked to control for possible modifications. Periodically, the absorption spectra and the fluorescence excitation and emission spectra were recorded.

The $\mathrm{Se}^{2-}$ ion contribution to the formation of nanoparticles may come from two different routes. One of them involves the decomposition in basic medium of selenosulphate in two stages:

$$
\begin{gathered}
\mathrm{SeSO}_{3}^{2-}+\mathrm{OH}^{-} \leftrightarrows \mathrm{HSe}^{-}+\mathrm{SO}_{4}^{2-} \\
\mathrm{HSe}^{-}+\mathrm{OH}^{-} \leftrightarrows \mathrm{Se}^{2-}+\mathrm{H}_{2} \mathrm{O}
\end{gathered}
$$

The second one involves the hydrolysis in basic medium of a complex formed by $\mathrm{Cd}^{2+}$ and selenosulphate:

$$
\begin{gathered}
2 \mathrm{SeSO}_{3}{ }^{2-}+\mathrm{Cd}^{2+} \leftrightarrows\left[\mathrm{Cd}\left(\mathrm{SeSO}_{3}\right)_{2}\right]^{2-} \\
{\left[\mathrm{Cd}\left(\mathrm{SeSO}_{3}\right)_{2}\right]^{2-}+2 \mathrm{OH}^{-}} \\
\longrightarrow \mathrm{CdSe}+\mathrm{SeSO}_{3}{ }^{2-}+\mathrm{SO}_{4}{ }^{2-}+\mathrm{H}_{2} \mathrm{O}
\end{gathered}
$$

Either way, or both, the fact is that the formation of nanoparticles is controlled by a slow kinetic chemical process similar to homogeneous phase precipitation processes.

The morphology of the CdSe nanoparticles was studied with TEM (Figure 1).
2.3.3. Absorption Spectrum. It is possible to obtain the mean value of the diameter of QDs by applying the Henglein equation (5), which relates it to the extrapolated wavelength, $\lambda_{e}$, and it is obtained by tracing the tangent to the absorption curve on the ascending segment, and it is clearly differentiated from the zero absorbance value:

$$
2 \bar{R}=\bar{d}=\frac{0.1}{\left(0.138-0.0002345 \cdot \lambda_{e}\right)} .
$$

Figure 2 shows the procedure. Obtaining the value of $\lambda_{e}$ is simple for times exceeding $95 \mathrm{~min}$, when there is no turbidity in the solution, and above $500 \mathrm{~nm}$ the base line coincides with $A=0$, but for lower times turbidity alters the baseline so the $\lambda_{e}$ value must be obtained from the cut between the line tangent to the ascending part of the spectrum and the constant baseline drawn by the value of absorbance at $600 \mathrm{~nm}$, as shown in Figure 2(a).

There is also a procedure for calculating the concentration of nanoparticles from the absorption spectral data. This method involves obtaining data by studying the region of wavelengths where the nanoparticles do not absorb radiation for excitonic processes and behave merely as colloids, dispersing the radiation as a function of size. In this region of the spectrum, the molar absorption coefficient of the nanoparticles, $\varepsilon_{\mathrm{CdS}}$, is independent of their size.

The concentration of colloidal particles and thus nanoparticles can be determined by measuring absorbance at $350 \mathrm{~nm}$ (or lower wavelengths) as an indirect measurement of scattered light, using a relationship between two variables that is dependent on the size. This relationship, previously used by other researchers $[13,14]$, which is derived empirically and is related to the Rayleigh expression (intensity of light scattered by colloidal particles with a diameter less than the wavelength of the incident light $\lambda$ ) is expressed in the following:

$$
C_{\mathrm{CdSe}}=\frac{A_{350}}{1.438 \times 10^{29} \cdot(2 R)^{3}},
$$

where $C_{\mathrm{CdSe}}$ is the concentration of nanoparticles in moles $L^{-1} ; A_{350}$ is the absorbance value measured at $350 \mathrm{~nm}$; and $R$ is the radius of the nanoparticle with a spherical shape.

Assuming the spherical shape of CdSe nanoparticles and taking into account their diameter the number of molecules that constitute a single CdSe nanocrystal at each instant can be calculated with the following expression:

$$
N_{\mathrm{CdSe}}=\frac{4 \Pi R^{3}}{3} \cdot \frac{\rho_{\mathrm{CdSe}} \cdot N_{0}}{M_{\mathrm{CdSe}}},
$$

where $N_{\text {CdSe }}$ is the number of CdSe molecules on each nanoparticle; $R$ is the radius of the NP in centimeters; $\rho_{\text {CdSe }}$ is the density of CdSe in $\mathrm{g} \mathrm{cm}^{-3} ; N_{0}$ is the Avogadro constant $\left(6.023 \times 10^{23} \mathrm{~mol}^{-1}\right)$, and $M$ is the molecular weight of CdSe $\left(191.36 \mathrm{~g} \mathrm{~mol}^{-1}\right)$. 


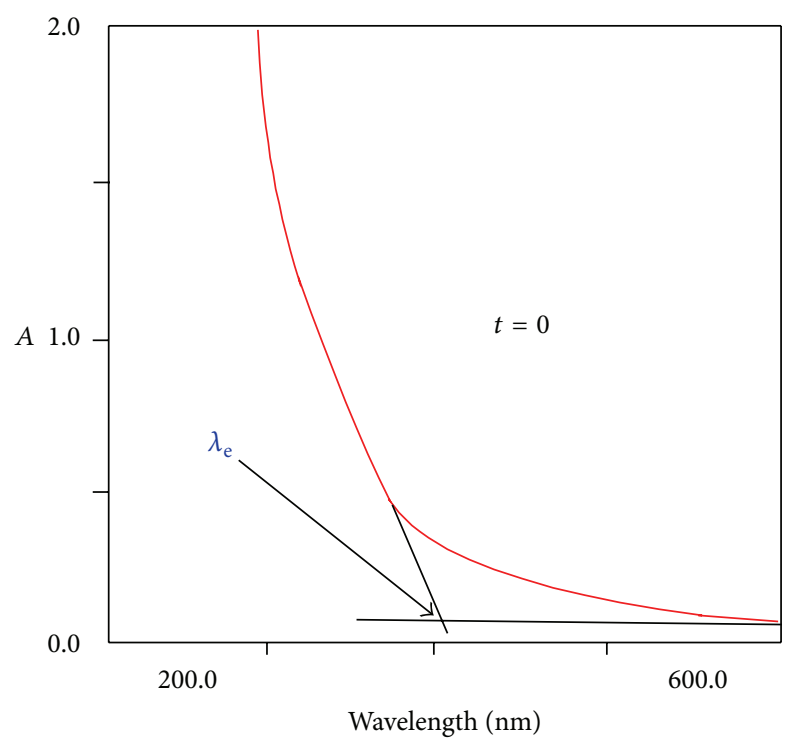

(a)

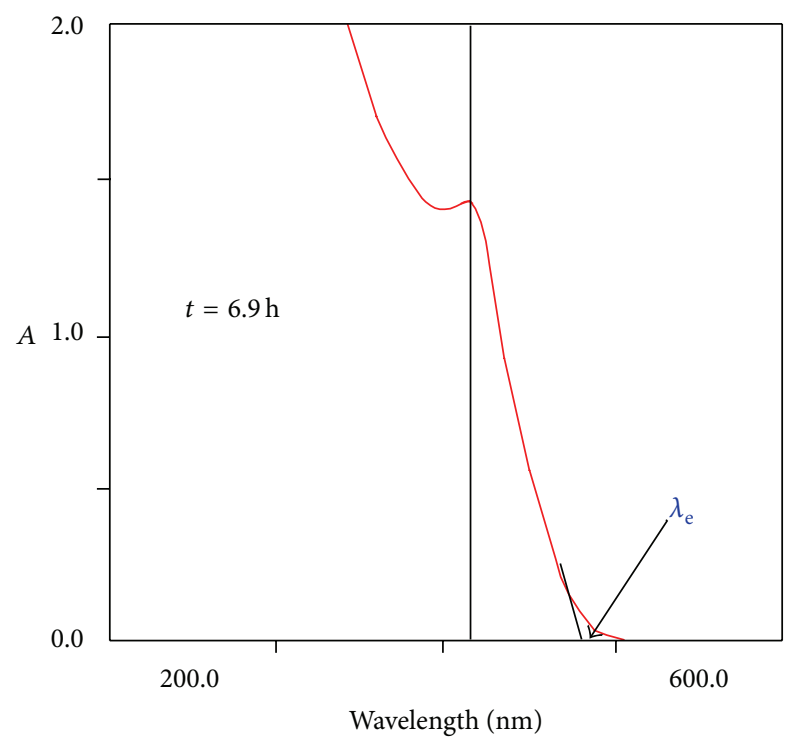

(b)

Figure 2: Absorption spectra of the CdSe quantum dots in aqueous solution. Calculating the value $\lambda_{e}$ for a cloudy solution (a) and a solution without turbidity (b).

By multiplying (6) and (7) it is possible to obtain an equation to calculate the molar concentration of CdSe forming the nanoparticles:

$$
\begin{aligned}
(6) \cdot(7) & =[\mathrm{CdSe}] \\
& =\frac{A_{350}}{1.438 \times 10^{29} \cdot R^{3}} \frac{4 \Pi R^{3}}{3} \frac{\rho_{\mathrm{CdSe}} \cdot N_{0}}{M_{\mathrm{CdSe}}} \\
& =0.667 \times 10^{-4} M A_{350} .
\end{aligned}
$$

2.3.4. Luminescence Spectrum. An important aspect of nanoparticles is their luminescence behavior. To study this feature, once the addition of $\mathrm{Na}_{2} \mathrm{SeSO}_{3}$ is finished, aliquots of the solution were collected and placed in the spectrofluorometer. The shape of the excitation spectrum affords information about the state of the surface of the nanoparticles and its changes with time. The emission spectrum provides information about the fluorescence emission intensity of the nanoparticle and the size distribution of nanoparticles around the average diameter $(\mathrm{d})$, which is related to the full width at half maximum (FWHM), also called $\Delta$.

Based on measurement of the parameters mentioned above, a detailed study of the effect of several variables on the nanoparticle collection process was performed. $\mathrm{N}_{2}$ must be bubbled through the solution prior to the addition of $\mathrm{SeSO}_{3}{ }^{2-}$. If this is performed during the formation of the CdSe nanoparticles, their size would increase and therefore their concentration in solution would reduce and, as a result, the fluorescence emission intensity would also decline. The rate of addition of the $\mathrm{SeSO}_{3}{ }^{2-}$ solution also affects the size of the nanoparticles: the higher this rate, the greater the size. Maintaining stirring after the addition of $\mathrm{SeSO}_{3}{ }^{2-}$ modifies the composition of the nanoparticle surface, adversely affecting their luminescence properties. Besides depending on the concentration of nanoparticles, the fluorescence emission intensity depends on a process that occurs simultaneously at the surface. This process may involve the formation of hydroxylated complexes of cadmium, the formation of a film or nanoshell of cadmium hydroxide, or both, all of them affecting the luminescence quantum yield. Maximum fluorescence intensity is reached when the thickness of the nanoshell reaches a specific value, related to the mean diameter of the nanoparticles, there being an optimum ratio between nanoshell size and the size of the nanoparticle.

\section{Results and Discussion}

A profound knowledge of all the variables allows choice of the optimal conditions necessary to achieve the desired property in the nanoparticles for a particular application. Thus, what type of nanoparticles are we looking for?

In some cases, highly stable nanoparticles, preserved in their own solution, will be desirable. In other cases, highly fluorescent nanoparticles will be what we are interested in. Finally, nanoparticles with a size as small as possible will find other applications. Here we describe three synthesis methods that achieve these three objectives individually.

3.1. Synthesis of Highly Stable CdSe Nanoparticles. In this case, the specific experimental conditions were as follows: $8 \mathrm{~mL}$ of $5 \times 10^{-2} \mathrm{M} \mathrm{CdCl}_{2}$ was diluted with bidistilled water up to $100 \mathrm{~mL}$. Then, $160 \mu \mathrm{L}$ of mercaptoacetic acid solution $1.41 \mathrm{M}$ was added. The $\mathrm{pH}$ of the solution was adjusted to 4.6 with $\mathrm{NaOH}$ and the flask was placed in an ice-bath until the solution reached $4^{\circ} \mathrm{C}$. At this point, $\mathrm{N}_{2}$ was bubbled through for $15 \mathrm{~min}$ with constant stirring, after which $2.5 \mathrm{~mL}$ of $0.0892 \mathrm{M} \mathrm{SeSO}_{3}{ }^{2-}$ was added over another $15 \mathrm{~min}$. The solution obtained was stored at $4^{\circ} \mathrm{C}$. Under these conditions, the final molar ratios were $\left[\mathrm{Cd}^{2+}\right]_{\mathrm{F}} /\left[\mathrm{Se}^{2-}\right]_{\mathrm{F}}=1.79 ;\left[\mathrm{Cd}^{2+}\right]_{\mathrm{F}} /[\mathrm{MAA}]_{\mathrm{F}}=1.77$. The 


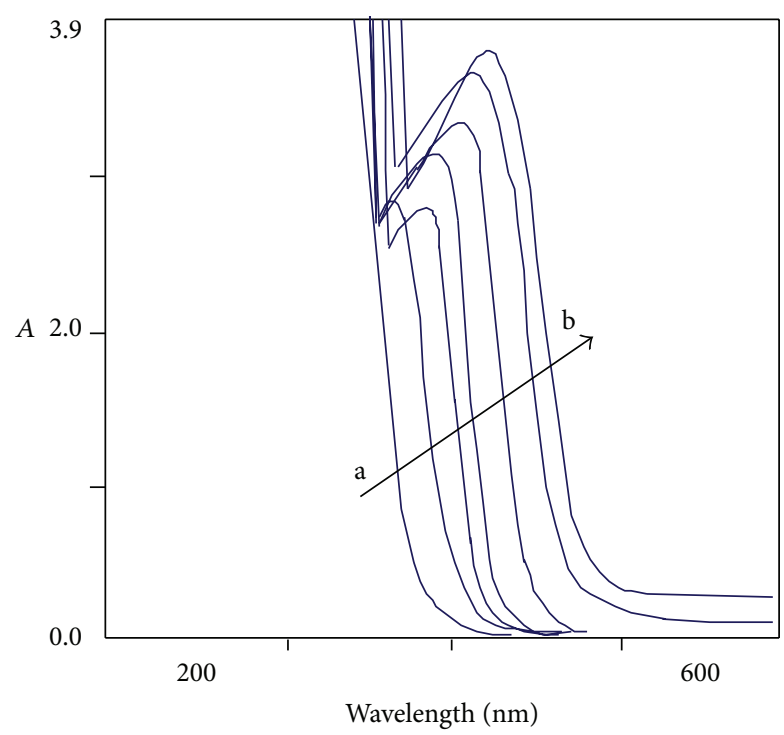

FIGURE 3: Evolution with time of the absorption spectrum of the highly stable CdSe QDs. From a $(t=0)$ to $\mathrm{b}(t=114$ days $)$.

absorption spectra were measured at room temperature and are plotted in Figure 3.

Figure 3 shows the absorption spectrum of the aqueous solution from which the nanoparticles were obtained and its evolution with time. The appearance of an absorption band can be seen, manifesting initially with an inflection zone at around $350 \mathrm{~nm}$ and thereafter evolving to a clear peak at $443.5 \mathrm{~nm}$ at 22 hours. This absorption band is due to the presence of CdSe nanoparticles in which the electrons are confined quantically, being able to absorb electromagnetic waves of a given energy to perform electronic transitions through the hollow bands of the semiconductor. With time, the absorption band is displaced towards higher wavelength values and the absorption value of the maximum of the band does so in the same sense. Both phenomena are related to the fact that the CdSe nanoparticles evolve with time, as seen from the increase in their mean diameter and in their concentration in solution.

The mean diameter changed from $2.1 \mathrm{~nm}$ at $t=0$ to $2.9 \mathrm{~nm}$ at $t=6$ days (Figure 4). This slow evolution was mainly due to kinetic control of the growth rate by the hydrolysis process at $4^{\circ} \mathrm{C}$. Following this, there was a period of more than 70 days during which the CdSe nanoparticles maintained a fairly constant size. From day 103 the presence of a small yellow precipitate was observed, and when the solution was homogenized the absorbance value at the baseline was increased. This phenomenon is due to the rearrangement of nanoparticles to create insoluble aggregates, a process that leads to an increase in nanoparticle size. The fluorescence intensity values are plotted against time in Figure 4.

As can be seen, the maximum value of fluorescence intensity was reached on day 29 and remained practically constant until about day 90 , thereafter starting to decline. In addition to their stability over a long period of time the CdSe

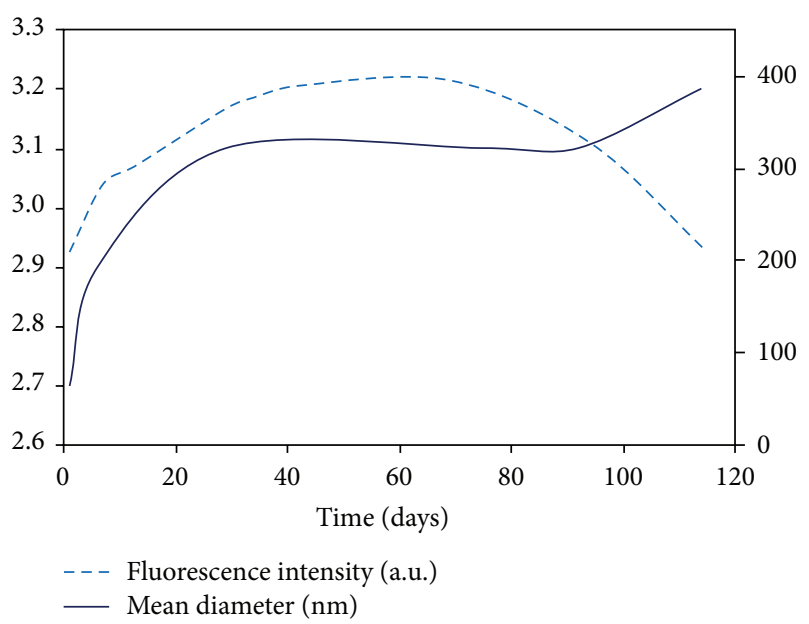

FIGURE 4: Evolution with time of the mean diameter (bold line) and the luminescence intensity (broken line) of the highly stable CdSe NPs. $\left[\mathrm{Cd}^{2+}\right]_{\mathrm{F}} /\left[\mathrm{Se}^{2-}\right]_{\mathrm{F}}=1.79 ;\left[\mathrm{Cd}^{2+}\right]_{\mathrm{F}} /[\mathrm{MAA}]_{\mathrm{F}}=1.77, \mathrm{pH}=4.6$; temperature: $4^{\circ} \mathrm{C} ;\left[\mathrm{Cd}^{2+}\right]_{\mathrm{F}}=3.9 \times 10^{-3}$; rendija: $3 \mathrm{~nm}$.

nanoparticles obtained under these experimental conditions had a relatively high and perfectly measurable fluorescence.

3.2. Obtaining Highly Fluorescent CdSe Nanoparticles. One of the possible applications of CdSe nanoparticles is their use in in vivo studies as carriers of molecules of biochemical interest, in which case their effectiveness must be followed through their luminescence properties. To ensure the sensitivity of the measurements it is useful to have highly fluorescent nanoparticles.

In this part of the study, the same synthesis procedure as that described above was followed, with one difference: the final concentration of $\mathrm{SeSO}_{3}{ }^{2-}$ was $1.06 \times 10^{-3} \mathrm{M}$. The molar ratios of the reagents were as follows: $\left[\mathrm{Cd}^{2+}\right]_{\mathrm{F}} /\left[\mathrm{Se}^{2-}\right]_{\mathrm{F}}=3.74$; $\left[\mathrm{Cd}^{2+}\right]_{\mathrm{F}} /[\mathrm{MAA}]_{\mathrm{F}}=1.77$. Because of the excess of $\mathrm{Cd}^{2+}$ ions in solution (compared to selenium ions) in this case the nucleation rate was higher than the growth rate.

The aim of this experiment was to obtain CdSe nanoparticles that would be as fluorescent as possible and would retain this property for as long as possible. Accordingly, below we focus our comments on the luminescence properties of nanoparticles obtained.

As can be seen in Figure 5, these nanoparticles had a very high fluorescence intensity, which remained practically constant between days 22 and 37. It was at this time when the optimal nanoparticle size/nanoshell size ratio was reached and when the quantum efficiency of the luminescence process was maximum.

3.3. Preparation of the Smallest Nanoparticles Possible. Size is an important factor in the ability of nanoparticles to cross the cell membrane and be effective in their role as drug carriers. When desired, by controlling the experimental conditions it is possible to obtain small nanoparticles that will remain small for long periods of time. As shown previously, the growth rate of nanoparticles can be controlled kinetically 


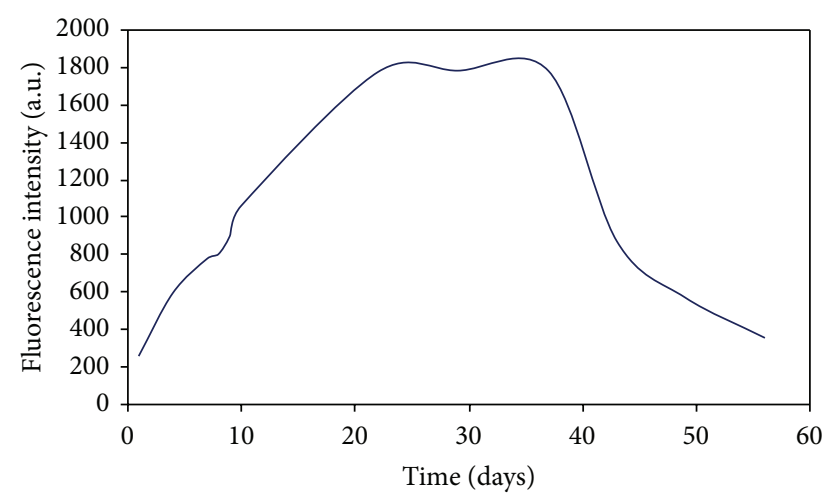

FIGURE 5: Evolution with time of the luminescence intensity of the highly fluorescent CdSe nanoparticles: $\left[\mathrm{Cd}^{2+}\right]_{\mathrm{F}} /\left[\mathrm{Se}^{2-}\right]_{\mathrm{F}}=3.74$; $\left[\mathrm{Cd}^{2+}\right]_{\mathrm{F}} /[\mathrm{MAA}]_{\mathrm{F}}=1.77, \mathrm{pH}=4.6$; temperature: $4^{\circ} \mathrm{C} ;\left[\mathrm{Cd}^{2+}\right]_{\mathrm{F}}=$ $3.9 \times 10^{-3}$; rendija: $3 \mathrm{~nm}$.

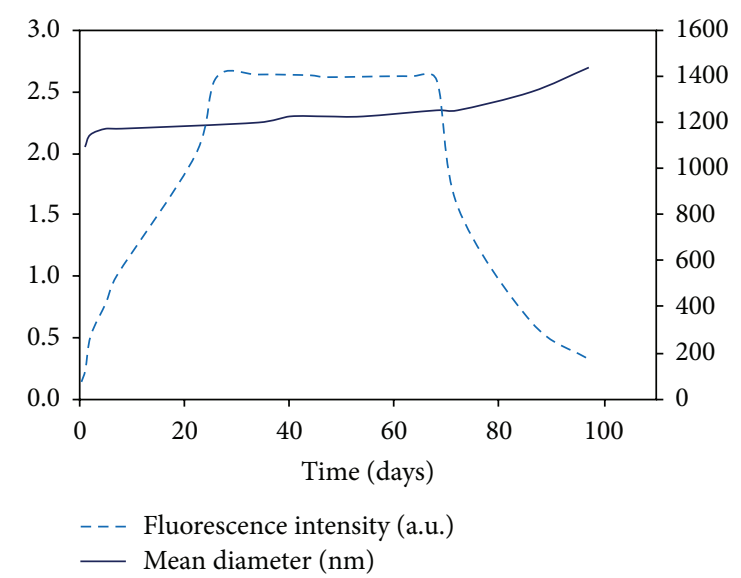

FIGURE 6: Evolution with time of the mean diameter (bold line) and the luminescence intensity (broken line) of the smallest CdSe nanoparticles possible: $\left[\mathrm{Cd}^{2+}\right]_{\mathrm{F}} /\left[\mathrm{Se}^{2-}\right]_{\mathrm{F}}=5.60 ;\left[\mathrm{Cd}^{2+}\right]_{\mathrm{F}} /[\mathrm{MAA}]_{\mathrm{F}}=$ $1.77, \mathrm{pH}=4.6$; temperature: $4^{\circ} \mathrm{C} ;\left[\mathrm{Cd}^{2+}\right]_{\mathrm{F}}=3.9 \times 10^{-3}$; rendija: $3 \mathrm{~nm}$.

by fixing the synthesis temperature at $4^{\circ} \mathrm{C}$ and the solution $\mathrm{pH}$ at 4.6. Additionally, the greater the excess of $\mathrm{Cd}^{2+}$ in solution over $\mathrm{SeSO}_{3}{ }^{2-}$, the higher the nucleation rate and the lower the growth rate. To check these theoretical aspects, CdSe nanoparticles were obtained under the experimental conditions of the two previous assays, but with the following molar ratios: $\left[\mathrm{Cd}^{2+}\right]_{\mathrm{F}} /\left[\mathrm{Se}^{2-}\right]_{\mathrm{F}}=5.60 ;\left[\mathrm{Cd}^{2+}\right]_{\mathrm{F}} /[\mathrm{MAA}]_{\mathrm{F}}=$ 1.77. The size of CdSe nanoparticles increased from $1.9 \mathrm{~nm}$ at $t=0$ to $2.2 \mathrm{~nm}$ at 5 days, but then their size remained smaller than $2.3 \mathrm{~nm}$ for more than 60 days (Figure 6).

Figure 6 shows that the maximum value of fluorescence intensity was reached at about the same time as in the previous experiment. In this experiment, the maximum fluorescence value was slightly lower but remained constant over a longer period of time. This difference can be attributed to the amount of mercaptoacetate surrounding the nanoparticles, depending on their size and affecting the stability of the colloidal system. Apparently, the number of MAA molecules
TABLE 1: Spectral characteristics of the CdSe nanoparticles from two different experiments performed under the same experimental conditions (Experiment A: bold; Experiment B, 23 h later: not bold).

\begin{tabular}{lccccccc}
\hline Time & $A$ & $\lambda_{\mathrm{abs}}$ & $d$ & $\mathrm{FI}$ & $\lambda_{\mathrm{ex}}$ & $\lambda_{\mathrm{em}}$ & \\
\hline \multirow{2}{*}{ 1 hour } & $\mathbf{2 . 5 0 3}$ & $\mathbf{3 6 2}$ & $\mathbf{2 . 2}$ & $\mathbf{2 7 . 6 4}$ & $\mathbf{3 9 5}$ & $\mathbf{4 9 2}$ & $\mathrm{A}$ \\
& 2.489 & 360 & 2.2 & 22.52 & 401 & 491 & $\mathrm{~B}$ \\
\hline \multirow{2}{*}{12 days } & $\mathbf{2 . 8 2 6}$ & $\mathbf{3 8 8}$ & $\mathbf{2 . 7}$ & $\mathbf{1 6 8 . 6 4}$ & $\mathbf{4 2 5}$ & $\mathbf{4 9 7}$ & $\mathrm{A}$ \\
& 2.853 & 393 & 2.7 & 172.73 & 425 & 497 & $\mathrm{~B}$ \\
\hline \multirow{2}{*}{62 days } & $\mathbf{2 . 9 3 7}$ & $\mathbf{3 9 8}$ & $\mathbf{2 . 8}$ & $\mathbf{9 4 . 9 1}$ & $\mathbf{4 3 2}$ & $\mathbf{5 0 2}$ & $\mathrm{A}$ \\
& 2.940 & 402 & 2.8 & 113.28 & 438 & 505 & $\mathrm{~B}$ \\
\hline
\end{tabular}

A: absorption; $\lambda_{\text {abs }}$ : absorption wavelength in $\mathrm{nm}$; $d$ : mean diameter in $\mathrm{nm}$; FI: fluorescence intensity in arbitrary units; $\lambda_{\text {ex }}$ : excitation wavelength in $\mathrm{nm}$; $\lambda_{\mathrm{em}}$ : emission wavelength in $\mathrm{nm}$.

surrounding one nanoparticle was higher in this experiment (because of the lower mean diameter), and hence stability was also higher.

3.4. Reproducibility in the Synthesis Process. One of the strongest criticisms usually made concerning the methods used for obtaining crystals, colloids, and nanoparticles is the lack of reproducibility due to the amount of variables that influence their synthesis, some of them uncontrolled. In order to check the reproducibility of the proposed method for the synthesis of CdSe nanoparticles in aqueous media, two experiments (A and B) were prepared and carried out under the same experimental conditions, but one of them $23 \mathrm{~h}$ later (B).

After studying the absorption spectra, it was found that there were no significant differences between the spectra of either experiment at similar times (Table 1). The size of the nanoparticles, the wavelength at which the inflection points appeared, and the maximum absorption value were similar for similar times.

However, reproducibility in the size and concentration of the $C_{\mathrm{CdSe}}$ nanoparticles obtained in two similar and parallel experiences is not sufficient to demonstrate the reproducibility of the method of synthesis. As demonstrated previously, the state of the surface of the nanoparticles is critical for explaining the luminescence properties. The only way to check that the surface is reproducible is to record the luminescence properties of the nanoparticles. By comparing the emission spectra of the nanoparticles for similar times, it is possible to check the reproducibility of the surface. Neither the optimal $\lambda_{\mathrm{em}}$ nor the value of fluorescence intensity differed significantly (Table 1).

The proposed method for preparing CdSe nanoparticles in aqueous medium showed good reproducibility.

\section{Conclusions}

We have developed a simple, easy, and cheap method for obtaining CdSe nanoparticles. Synthesis is performed in an aqueous medium and this solves one of the drawbacks of synthesis in organic media, namely, the later stage of solubilization in aqueous media, which sometimes alters not 
only the size of the nanoparticles but also their luminescence properties. By controlling the experimental conditions, CdSe quantum dots of sizes ranging between 2 and $6 \mathrm{~nm}$ can be obtained.

We achieved a very slow nanocrystal growth rate due to the kinetic control of the low temperature $\left(4^{\circ} \mathrm{C}\right)$, which controls $\mathrm{SeSO}_{3}{ }^{2-}$ hydrolysis to generate $\mathrm{Se}^{2-}$. Thus, the method can be referred to as the in situ and homogeneous phase generation of nanocrystal-forming $\mathrm{Se}^{2-}$ species.

\section{Conflict of Interests}

The authors declare that there is no conflict of interests regarding the publication of this paper.

\section{Acknowledgment}

The authors thank the Council for Education and Culture of the Regional Government of Castilla y León for financial support.

\section{References}

[1] D. Ramadurai, D. Geerpuram, D. Alexson et al., "Electrical and optical properties of colloidal semiconductor nanocrystals in aqueous environments," Superlattices and Microstructures, vol. 40, no. 1, pp. 38-44, 2006.

[2] S. Pathak, M. C. Davidson, and G. A. Silva, "Characterization of the functional binding properties of antibody conjugated quantum dots," Nano Letters, vol. 7, no. 7, pp. 1839-1845, 2007.

[3] R. S. Chouhan, A. C. Vinayaka, and M. S. Thakur, "Thiolstabilized luminescent CdTe quantum dot as biological fluorescent probe for sensitive detection of methyl parathion by a fluoroimmunochromatographic technique," Analytical and Bioanalytical Chemistry, vol. 397, no. 4, pp. 1467-1475, 2010.

[4] W. W. Yu, L. Qu, W. Guo, and X. Peng, "Experimental determination of extinction coefficient of CdTe, CdSe, and CdS nanocrystals," Chemical Materials, vol. 15, no. 14, pp. 2854-2860, 2003.

[5] A. P. Alivisatos, "Semiconductor clusters, nanocrystals, and quantum dots," Science, vol. 271, no. 5251, pp. 933-937, 1996.

[6] C. J. Murphy and J. L. Coffer, "Quantum dots: a primer," Applied Spectroscopy, vol. 56, no. 1, pp. 16A-27A, 2002.

[7] W. W. Yu, E. Chang, R. Drezek, and V. L. Colvin, "Water-soluble quantum dots for biomedical applications," Biochemical and Biophysical Research Communications, vol. 348, no. 3, pp. 781786, 2006.

[8] F. Pinaud, X. Michalet, L. A. Bentolila et al., "Advances in fluorescence imaging with quantum dot bio-probes," Biomaterials, vol. 27, no. 9, pp. 1679-1687, 2006.

[9] T. Jamieson, R. Bakhshi, D. Petrova, R. Pocock, M. Imani, and A. M. Seifalian, "Biological applications of quantum dots," Biomaterials, vol. 28, no. 31, pp. 4717-4732, 2007.

[10] P. Alivisatos, "The use of nanocrystals in biological detection," Nature Biotechnology, vol. 22, no. 1, pp. 47-52, 2004.

[11] P. T. Tran, E. R. Goldman, G. P. Anderson, J. M. Mauro, and H. Mattoussi, "Use of luminescent CdSe-ZnS nanocrystal bioconjugates in quantum dot-based nanosensors," Physica Status Solidi (B), vol. 229, pp. 427-432, 2002.
[12] D. Gerion, F. Pinaud, S. C. Williams et al., "Synthesis and properties of biocompatible water-soluble silica-coated CdSe/ZnS semiconductor quantum dots," Journal of Physical Chemistry B, vol. 105, no. 37, pp. 8861-8871, 2001.

[13] H. Zhang, Z. Cui, Y. Wang et al., "From water-soluble CdTe nanocrystals to fluorescent nanocrystal-polymer transparent composites using polymerizable surfactants," Advanced Materials, vol. 15, no. 10, pp. 777-780, 2003.

[14] J. Weng, X. Song, L. Li et al., "Highly luminescent CdTe quantum dots prepared in aqueous phase as an alternative fluorescent probe for cell imaging," Talanta, vol. 70, no. 2, pp. 397-402, 2006. 

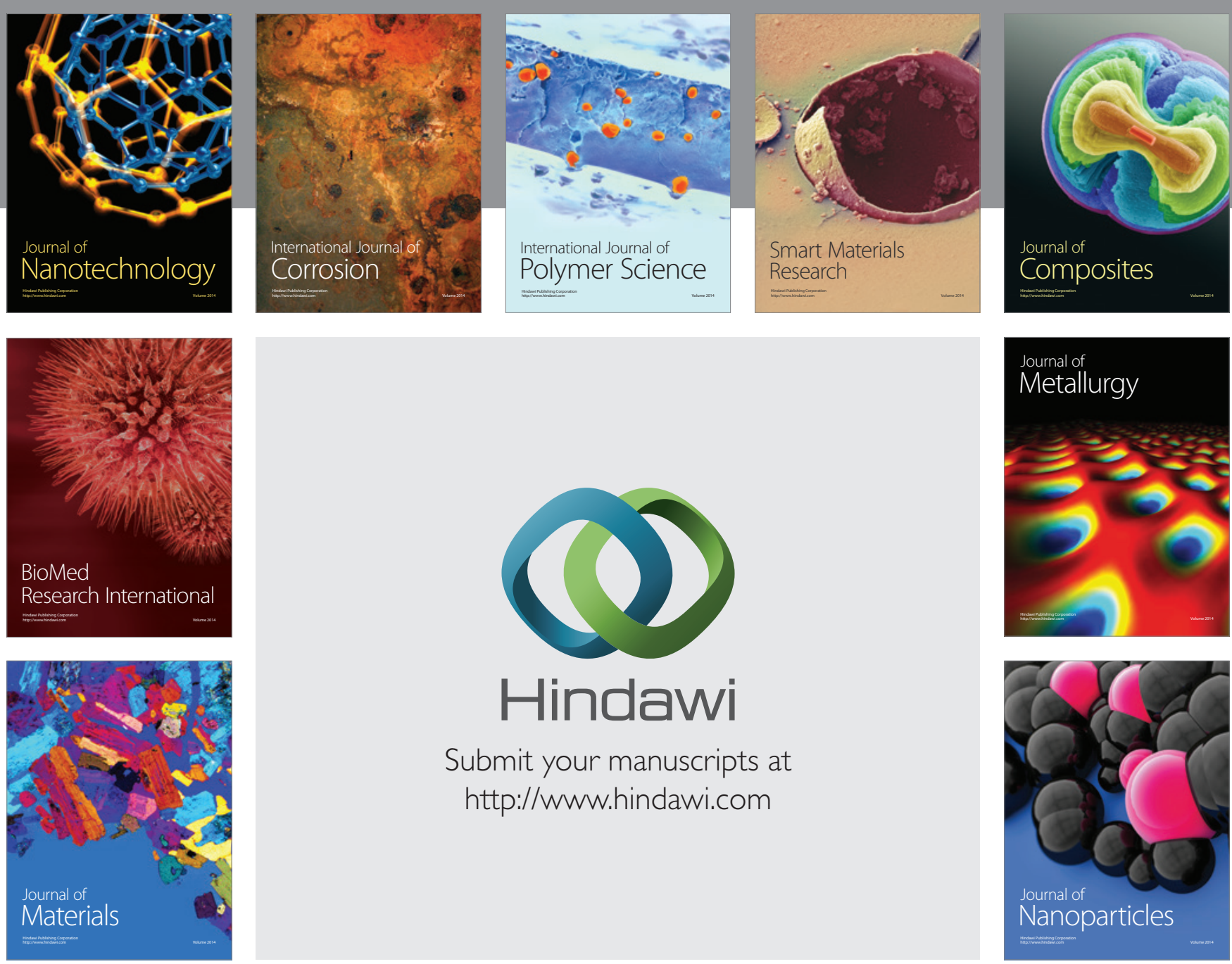

Submit your manuscripts at http://www.hindawi.com
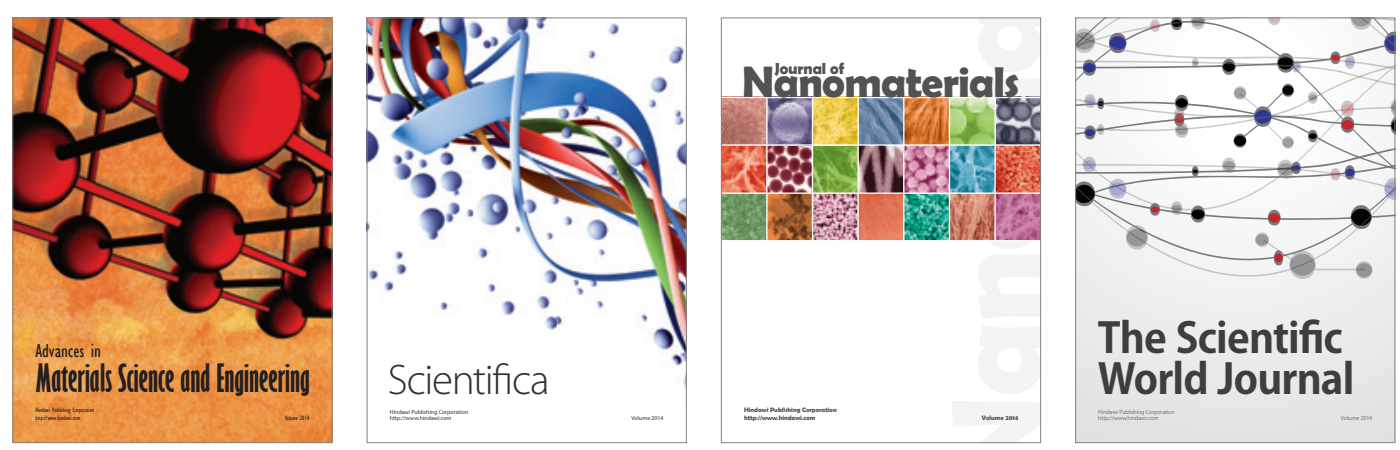

\section{The Scientific World Journal}
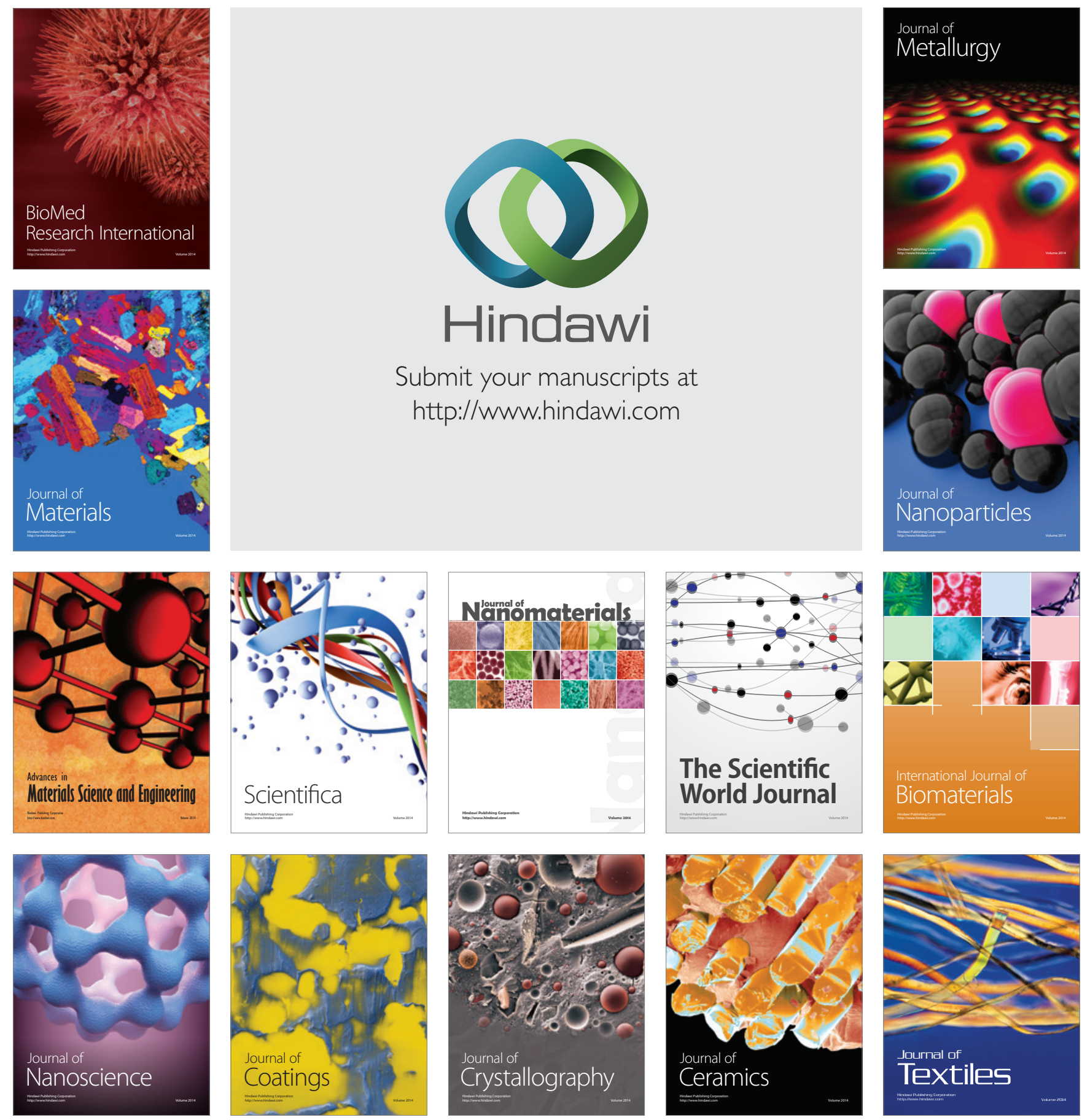